\title{
La similitud de relaciones como un rasgo definitorio de las analogías: Un cuestionamiento desde el enfoque categorial
}

\author{
Lucía Micaela Tavernini ${ }^{1}$ y Ricardo Adrián Minervino ${ }^{2}$
}

\begin{abstract}
RESUMEN
La teoría de proyección de la estructura postula que el componente central en la realización de una analogía es detectar que dos situaciones comparten un sistema de relaciones similares. Se llevaron a cabo dos experimentos con el objetivo de evaluar la hipótesis del enfoque categorial de que la similitud de relaciones no constituye una condición necesaria ni suficiente para que las personas juzguen que dos hechos son análogos, sino que el factor determinante es la pertenencia o no de los hechos comparados a una misma categoría relacional. En ambos experimentos 18 estudiantes de Psicologia recibieron un hecho base y dos hechos meta en los que se describian eventos simples, y debían evaluar cuán análogo al hecho base encontraban a cada hecho meta. En el Experimento 1 los dos hechos meta incluían relaciones que no eran similares a las del hecho base. Sin embargo, mientras que en el primer hecho meta el objeto al que se aplicaba la acción descrita por la relación convertía al hecho en un ejemplar de la categoría relacional aplicable al base, el objeto del segundo hecho meta lo convertía en un ejemplar de una categoria alternativa. El Experimento 2 tuvo la misma estructura que el primero, con la diferencia de que las relaciones base y meta eran similares. En ambos experimentos, los resultados mostraron que el primer hecho meta fue considerado análogo al hecho base, pero no el segundo. Esto evidenció que el parecido de relaciones no es necesario (Experimento 1) ni suficiente (Experimento 2) para considerar que dos hechos son análogos y que, en cambio, el criterio que emplean las personas para determinar que dos hechos son o no análogos es el de pertenencia categorial. Se discuten las implicaciones de estos hallazgos para la formulación de modelos teóricos y computacionales sobre el pensamiento analógico.
\end{abstract}

Palabras clave: analogia, categoria relacional, relaciones, similitud.

\section{Relational similarity as a defining feature of analogies: A questioning from the categorical approach}

\begin{abstract}
The structure-mapping theory posits that the central component in establishing an analogy is to discover that two situations share a system of similar relations. Two experiments were performed to evaluate the hypothesis of the categorical approach that relational similarity does not constitute a necessary nor a sufficient condition for people to judge that two facts are analogous, and that the determinant factor is whether or not
\end{abstract}

\footnotetext{
${ }^{1}$ Consejo Nacional de Investigaciones Ciéntificas y Técnicas (CONICET), Universidad Nacional del Comahue, Universidad Nacional de Córdoba, Argentina; micaelatavernini@gmail.com

2 Consejo Nacional de Investigaciones Ciéntificas y Técnicas (CONICET), Universidad Nacional del Comahue, Argentina.
}

\section{AGRADECIMIENTOS}

Este trabajo fue financiado por la Agencia Nacional de Promoción Científica y Técnológica (ANPCyT) a través del subsidio PICT 2016-0363, por la Universidad Nacional del Comahue a través de los subsidios B213, C123 y C134, y por el Consejo Nacional de Investigaciones Científicas y Técnicas (CONICET) a través del subsidio PIP 0576. 
the compared facts belong to a common schema-governed category. In both experiments, 20 psychology students received a base fact and two target facts describing simple events, and they had to evaluate how analogous the base and each of the target facts were. In Experiment 1 both target facts included relations that were not similar to those included in the base fact. However, while in the first target fact the object to which the action described by the relation was applied made the whole fact became an exemplar of the schema-governed category applicable to the base, the object of the second target fact made it became an exemplar of an alternative schema-governed category. Experiment 2 has the same structure than the first one, with the only difference being that base and target relations were similar. In both experiments results showed that the first target fact was considered analogous to the base fact, but not the second one. This evidenced that relational similarity is not necessary (Experiment 1) nor sufficient (Experiment 2) to consider that two facts are analogous, and that, instead, the criterion employed by people to determine that two facts are or are not analogous is category membership. We discussed the implications of these results for the development of theoretical and computational models on analogical thinking.

Keywords: analogy, schema-governed category, relations, similarity.

El pensamiento analógico desempeña un rol en actividades tan diversas como la solución de problemas, la argumentación, la explicación, el aprendizaje de categorias o la toma de decisiones (de la Fuente \& Minervino, 2008; Gentner \& Maravilla, 2018; Holyoak, 2012), por lo que no es extraño que se lo considere un componente central de la inteligencia (Gentner, 2003; Hofstadter \& Sander, 2013; Holyoak \& Thagard, 1995).

El razonamiento por analogía ha sido definido como la capacidad para detectar que dos hechos comparten un mismo patrón relacional, más allá de las diferencias existentes entre las entidades (individuos y objetos) que componen las situaciones comparadas (Goswami, 2001; Inhelder \& Piaget, 1955; Piaget, 1957; Richland, Morrison \& Holyoak, 2006; Sternberg \& Nigro, 1980; Raven, Raven \& Court, 1962). La teoría de proyección de la estructura (Falkenhainer, Forbus \& Gentner, 1989; Genter, 1983, 1989; Gentner \& Markman, 1997) es la teoría dominante en el campo desde los 80 a la actualidad y constituye uno de los casos más extremos de esta posición, ya que mientras algunas teorias (e.g., la teoría de las múltiples restricciones, Holyoak \& Thagard, 1989; Hummel \& Holyoak, 1997) han reconocido que el parecido de entidades juega un papel en determinar si dos hechos pueden ser considerados análogos, la teoría de proyección de la estructura entiende que, si las entidades son similares, no se trata de una analogía sino de otro tipo de comparación (Gentner, 1983, 1989). Esta teoría postula así que el parecido de relaciones constituye un rasgo definitorio de las analogias, en el sentido de que su presencia, junto a otros rasgos formales de las analogias, es clave para la existencia de una analogía.

En este trabajo se presentan los resultados de dos experimentos, desarrollados con el propósito de mostrar que el parecido de relaciones entre dos hechos no constituye una condición necesaria ni suficiente para que las personas juzguen que esos hechos son análogos. Esta tesis se sigue de un enfoque alternativo al enfoque estándar sobre el pensamiento analógico (Minervino, Oberholzer \& Trench, 2008, 2013; Minervino, Tavernini, Gómez \& Trench, 2017; Oberholzer, Trench \& Minervino, 2011; Tavernini, Trench, Olguín \& Minervino, en prensa), de acuerdo con el cual dos hechos son análogos si pueden ser considerados casos de una misma categoría relacional de esquema (CRE; Gentner \& Kurtz, 2005; Goldwater, Markman \& Stilwell, 
2011; Markman y Stilwell, 2001), algo que puede ocurrir en ausencia de similitud de relaciones (no necesidad), así como no tener lugar a pesar de la presencia de similitud de relaciones (no suficiencia). La pertenencia o no de un hecho a una CRE depende, de acuerdo con este enfoque, de las complejas interdependencias semánticas que mantienen los llenadores de los diversos roles temáticos (e.g., relaciones, agentes, pacientes, objetos o instrumentos) de una situación que definen la estructura de este tipo de categorias (Tavernini, Martín \& Minervino, 2017). Presentamos en primer lugar los postulados centrales de la teoría de proyección de la estructura de Gentner $(1983,1989)$ y luego las ideas centrales del enfoque categorial (Minervino et al., 2008, 2013), para presentar después los dos experimentos referidos.

\section{La teoría de proyección de la estructura}

La teoría de proyección de la estructura considera que realizar una analogia consiste en descubrir que dos situaciones, cuyas entidades no son parecidas, comparten una estructura de relaciones similares (Gentner, 1983, 1989; Gentner \& Markman, 1997). Una vez detectada esta estructura compartida, aquello que resulta conocido acerca de la situación más familiar (análogo base: $\mathrm{AB}$ ) puede ser utilizado para inferir nueva información sobre la situación menos conocida (análogo meta: AM). La búsqueda de una estructura compartida supone la aplicación del principio de sistematicidad (Gentner, 1983, 1989), a través del cual el sistema cognitivo promueve la puesta en correspondencia de relaciones de orden inferior vinculadas por relaciones de orden superior antes que el apareamiento de relaciones aisladas. La alineación entre los sistemas de relaciones puestos en correspondencia debe, además, satisfacer los principios de correspondencias uno-a-uno y conectividad paralela. Mientras que el establecimiento de correspondencias uno a uno requiere que un elemento de una situación sea puesto en correspondencia con, como máximo, un elemento de la otra situación, la conectividad paralela implica que, si dos predicados son puestos en correspondencia, sus argumentos deben también ser apareados, de acuerdo a los roles que cumplen (e.g., agente, paciente, instrumento u objeto). Supóngase que una persona debe comparar las siguientes situaciones:

AB: Juan, que juega al fútbol, ama a María, y esto hace que Juan se preocupe por Maria.

AM: El perro, que se entretiene con los ovillos de lana, adora los huesos de asado.

Es posible establecer dos grupos de correspondencias entre estas situaciones: (a) aquél que pone en correspondencia las relaciones jugar y entretenerse y sus argumentos (Juan y perro, en tanto agentes de dichas acciones, y fútbol y ovillos de lana, en tanto objetos a los que se aplican las acciones), y (b) aquél que pone en correspondencia amar y adorar y sus argumentos (Juan y perro, y Maria y huesos de asado). Una vez que se han establecido las correspondencias entre el AB y el AM, las afirmaciones del AB que están conectadas a un grupo de elementos puestos en correspondencia, pero que no poseen una contraparte en el AM, serán propuestas como inferencias candidatas a través de un proceso de copia con sustitución y generación (Gentner, 1989). En este ejemplo, el sistema cognitivo podría transferir la proposición base "esto hace que Juan se preocupe por María" al 
meta, copiando la relación preocuparse-por y reemplazando Juan por perro y María por huesos de asado, tal como es indicado por las correspondencias previamente establecidas. La interpretación de la analogía que incluye esta inferencia será preferida por sobre la alternativa en virtud del principio de sistematicidad, el que busca favorecer correspondencias con potencia inferencial (Clement \& Gentner, 1991; Gentner, Ratterman \& Forbus, 1993).

Una tesis central de la teoria de la proyección de la estructura es que dos relaciones pueden ser puestas en correspondencia únicamente si son idénticas o si admiten ser re-representadas en términos idénticos en un nivel superior relativamente inmediato. Esta re-representación puede ser lograda por medio de la búsqueda de un término supraordenado común relativamente inmediato (Falkenhainer, 1990), o por medio de la descomposición del significado de las relaciones en sus subcomponentes semánticos (Yan, Forbus $\&$ Gentner, 2003). Por ejemplo, en la analogía presentada anteriormente amar y adorar pueden ser re-representados como mantener una emoción positiva. De acuerdo con la teoría de la proyección de la estructura, el juicio de las personas acerca de la calidad de una analogía depende del número de correspondencias locales, de la profundidad del sistema de correspondencias y -de particular interés para el presente estudio- del grado de similitud que mantienen las relaciones puestas en correspondencia (Forbus \& Gentner, 1989; Gentner, 1983; Gentner et al., 1993). Es importante aclarar que, de acuerdo con esta teoría, si dos relaciones mantienen un parecido en un nivel muy abstracto y trivial de representación (e.g., amar y comer son dos actividades) el sistema cognitivo no admite que sean apareadas y, por lo tanto, juzga como no análogas las situaciones de las que estas relaciones forman parte (Gentner \& Markman, 2005). En tanto el parecido de entidades no cuenta en absoluto para que dos hechos sean considerados análogos (e.g., el hecho de que Maria y huesos de asado sean diferentes no hace que los hechos no sean análogos), la similitud de relaciones constituye, por lo que respecta al papel de la semántica en el razonamiento analógico -junto a los requisitos formales descritos- una condición necesaria y suficiente (i.e, un componente definitorio) para que dos hechos sean vistos como análogos.

\section{El enfoque categorial}

Contrariamente a los ejemplares de las categorias de entidades (e.g., fruta), los que comparten un conjunto de rasgos probabilísticos (e.g., ser comestible, ser dulce o tener cáscara) y de correlaciones entre estos rasgos, los miembros de una CRE (e.g., seducción) comparten una estructura (e.g., son casos en los que un agente realiza una acción hacia un paciente con la finalidad de manifestar su interés amoroso por ese paciente) que puede ser instanciada por ejemplares muy diferentes (Gentner \& Kurtz, 2005; Goldwater et al. 2011; Markman \& Stilwell, 2001). Por ejemplo, la CRE seducción puede ser instanciada por ejemplares como "El taxista le regaló un perfume a la pasajera", "El taxista le escribió un poema a la pasajera", "El taxista invitó a un baile a la pasajera" o "El taxista le alabó el color de ojos a la pasajera". Estos casos constituyen ejemplares de la categoría seducción a pesar de que no mantienen similitud en sus relaciones (e.g., regalar no es semánticamente similar a escribir). Sin embargo, hechos tales como "El taxista le dio una tarjeta de la empresa a la pasajera", "El taxista le anotó la dirección de un museo a la pasajera", "El taxista convidó a la pasajera un caramelo" o "El taxista le elogió la puntualidad a la pasajera", no constituyen casos de 
seducción a pesar de que las relaciones de cada uno de ellos son similares a uno de la serie anterior (e.g., dar es semánticamente similar a regalar). De esta forma, si una persona tuviera que decidir si "El taxista le regaló un perfume a la pasajera" y "El taxista le escribió un poema a la pasajera" son análogos, es altamente probable que diera una respuesta positiva, a pesar de que las relaciones regalar y escribir no son similares. En cambio, si tuviera que decidir si "El taxista le regaló un perfume a la pasajera" y "El taxista le dio una tarjeta de la empresa a la pasajera" son análogos es altamente probable que diera una respuesta negativa, a pesar de que las relaciones regalar y dar son similares. La explicación de estos hechos por parte del enfoque categorial consiste en que en el primer caso se trata de dos ejemplares de una misma CRE (seducción), mientras que en el segundo caso no (uno es un caso de seducción y el otro un caso de publicidad). El primer caso muestra que el parecido de relaciones no es necesario para que dos hechos sean considerados análogos, mientras que el segundo muestra que no es suficiente. De acuerdo al enfoque categorial, cuando una persona se enfrenta a la tarea de determinar si dos hechos son análogos, se preocupa por establecer si los hechos, interpretados como un todo, pueden ser considerados casos de una misma CRE y, por tanto, desatiende a la similitud local que pueden o no mantener las relaciones. Con respecto a la evaluación de calidad de una analogia, el enfoque de la asignación categorial postula que dos hechos serán considerados más o menos análogos en la medida en que exhiban valores más o menos cercanos en ciertas dimensiones de la CRE a la que ambos pertenecen. Por ejemplo, la osadia, el romanticismo, la originalidad o el carácter directo constituyen dimensiones salientes de la CRE seducción. Así, si dos actos de seducción son altamente románticos, serán considerados más análogos que si uno de ellos es poco romántico.

En este trabajo presentamos los resultados de dos experimentos. E1 objetivo del primero fue poner a prueba la hipótesis de que la similitud de relaciones no constituye una condición necesaria para que dos hechos sean considerados análogos, ya que si dos hechos no mantienen relaciones similares, pero pertenecen a una misma CRE, serán considerados de ese modo. El objetivo del segundo experimento consistió en evaluar la hipótesis de que la similitud de relaciones no es una condición suficiente para que dos hechos sean considerados análogos, ya que si dos hechos no constituyen ejemplares de una misma CRE no serán considerados análogos incluso si sus relaciones se parecen. En ambos experimentos, la pertenencia (o no pertenencia) de un hecho a una CRE estará determinada por la entidad a la que se aplica la relación en cada caso, entidad que cumple el rol temático de objeto.

\section{EXPERIMENTO 1}

El objetivo del primer experimento fue poner a prueba la hipótesis de que la similitud de relaciones no constituye una condición necesaria para que dos hechos sean considerados análogos. Esta hipótesis tiene su fundamento en la idea de que, aunque dos hechos no mantengan relaciones similares, pueden aún ser análogos si pertenecen a una misma CRE. Los participantes recibieron un hecho base seguido de dos hechos meta, con la instrucción de determinar el grado en que cada hecho meta podía ser considerado análogo al hecho base. Ninguno de los dos hechos meta se parecía al base por lo que respecta a sus relaciones. Sin embargo, uno de los hechos meta pertenecía a 
la misma CRE que el hecho base. Por ejemplo, el hecho base de uno de los conjuntos de materiales era "La policía arrojó gases a los manifestantes". Uno de los hechos meta de este conjunto era "La policía abrió las mochilas a los manifestantes". En este hecho meta, el objeto al que se aplica la acción descrita por la relación abrir (mochila), lo convierte en un ejemplar de la categoria represión, también aplicable al hecho base. El restante hecho meta de este conjunto de materiales era "La policía abrió la puerta a los manifestantes". La aplicación de abrir al objeto puerta no resulta en un caso de represión sino en uno de la CRE cortesía. La predicción que se sigue de la teoría de la proyección de la estructura es que los participantes considerarian que ninguno de los dos hechos meta es análogo al hecho base, por no haber similitud entre las relaciones de los hechos comparados. En cambio, la predicción del enfoque categorial es que, más allá de que no haya parecido entre las relaciones base y meta, las personas considerarian como análogos a los pares cuyos hechos pertenecian a una misma CRE y, en cambio, no considerarían como análogos a los pares cuyos hechos no podían ser asignados a una misma CRE. Si se dieran estos últimos resultados se contaría con una prueba de que es posible la existencia de analogías entre dos hechos que no mantienen relaciones similares, siendo clave para ello que la interacción entre la relación y los demás componentes de la situación resulten, en cada uno de los hechos comparados, en ejemplares de una misma CRE.

\section{MÉTODO}

\section{Participantes}

Formaron parte de este estudio 18 estudiantes de Psicología de la Universidad Nacional del Comahue $(M=20.12$ años; $D E=3.15)$. Todos los participantes firmaron un consentimiento informado antes de comenzar la experiencia.

\section{Diseño y procedimiento}

La variable independiente fue la pertenencia de los hechos comparados a una misma CRE (sí o no) y recibió una manipulación intrasujeto. La variable dependiente fueron los juicios sobre la calidad de las analogias. Los materiales fueron presentados por escrito. Los participantes fueron evaluados individualmente. Luego de que firmaran el consentimiento informado, recibieron un cuadernillo impreso compuesto por un instructivo, dos tareas de práctica y las tareas experimentales propiamente dichas. En cada tarea (de práctica y experimental) los participantes debian evaluar la calidad de las comparaciones a través de una escala Likert y luego justificar por escrito su respuesta. Luego de 5 minutos de trabajo para las dos tareas de práctica, los participantes podian plantear sus dudas con respecto a las mismas. No recibieron retroalimentación sobre sus respuestas, pero sí sobre el modo de realizar la tarea. Se les indicó luego que comenzaran con la tarea experimental. Se les dijo que esta consistiria en resolver seis tareas idénticas a las anteriores, y que solo cuando respondieran las preguntas para cada tarea podrian pasar a resolver la tarea siguiente. Se les aclaró que, si bien les llevaría aproximadamente 30 minutos resolver todas las tareas, no tendrían límite de tiempo alguno y podrian tomar descansos si los necesitaran. 


\section{Materiales}

El cuadernillo incluía un instructivo, dos tareas de práctica y seis tareas experimentales. Se les proporcionó a todos un lápiz y una goma de borrar. En la primera hoja del cuadernillo se ofrecía una descripción general de la tarea. En las siguientes dos hojas se presentaban las dos tareas de práctica (una por hoja), y a partir de la tercera hoja se presentaban las tareas experimentales (una por hoja). En cada tarea (de práctica y experimental) se presentó un hecho base centrado en la parte superior de la hoja (presentado como Hecho clave) y debajo de este, ubicados uno a la izquierda y otro a la derecha, dos hechos meta (presentados como Hecho 1 el de la izquierda y Hecho 2 el de la derecha). Cada hecho consistía en una oración que describía un evento simple. Debajo de cada uno de los correspondientes hechos meta aparecian las siguientes preguntas: 1) “¿En qué medida considerás que el hecho clave y el hecho 1 son análogos?”, y 2) “¿En qué medida considerás que el hecho clave y el hecho 2 son análogos?”. Para contestar estas preguntas, los participantes debian marcar su respuesta en una escala Likert de cinco puntos, en la que 1 era (Nada análogos), 2 (Apenas análogos), 3 (Medianamente análogos), 4 (Bastante análogos), y 5 (Totalmente análogos). Para justificar las puntuaciones dadas, los participantes debian completar las siguientes oraciones, que aparecian en la parte inferior de la hoja: 1) "Asigné esa puntuación a la comparación entre el hecho clave y el hecho 1 porque...", y 2) "Asigné esa puntuación a la comparación entre el hecho clave y el hecho 2 porque...".

Se construyeron seis conjuntos de materiales, cada uno de los cuales estuvo conformado por un hecho base y dos hechos meta. Uno de los conjuntos de materiales fue el siguiente:

Hecho clave. Juan le cortó el pasto a la anciana.

Hecho meta 1. Juan le llevó un perro de la calle a la anciana.

Hecho meta 2. Juan le llevó los medicamentos a la anciana.

Tal como lo ilustra este conjunto, los dos hechos meta incluian relaciones idénticas entre sí (llevar), pero que no eran similares a las de hecho base (cortar). Los hechos meta tampoco eran similares con respecto al base ni entre sí por lo que respecta al objeto al que se aplicaba la acción. Sin embargo, mientras que en uno de los hechos meta el objeto al que se aplicaba la acción descrita por la relación (perro de la calle) no lo convertía en un ejemplar de la CRE que es aplicable al hecho base (ayuda), en el segundo sí lo hacía (medicamentos). Los materiales tenían una estructura proposicional RELACION (agente, objeto, paciente) y los agentes y pacientes se mantenian constantes en los tres hechos. En la Tabla 1 se presentan tres conjuntos de materiales de los seis empleados. El orden de presentación de los materiales y la ubicación derecha-izquierda de los dos hechos meta fue decidido por azar simple para cada participante. El grado de similitud de las relaciones y entidades incluidas en los materiales de este experimento fue determinado por un grupo independiente de 20 participantes (extraídos de la misma población que los participantes experimentales). Se les pidió que puntuaran la similitud entre los elementos base y meta (e.g., la similitud entre cortar y llevar). Este grupo recibió una lista con pares de conceptos, algunos de los cuales eran pares de relaciones y otros pares de objetos, provenientes de un conjunto inicial de materiales. Debían emitir un juicio de similitud a través de una 
escala Likert de cinco puntos: 1 (No similares), 2 (Apenas similares), 3 (Medianamente similares), 4 (Muy similares), 5 (Totalmente similares). Se seleccionaron para formar parte de este experimento seis conjuntos de materiales cuyos pares de elementos (i.e., relaciones y objetos) obtuvieron una puntuación media de similitud inferior a 2.

Tabla 1.

Ejemplos de conjuntos de materiales empleados en el Experimento 1.

\begin{tabular}{cll}
\hline $\begin{array}{c}\text { Conjunto } \\
\mathbf{N}^{\circ}\end{array}$ & \multicolumn{1}{c}{ Hechos clave y meta } & \multicolumn{1}{c}{ CRE } \\
\hline \multirow{3}{*}{1} & HC. La policía les arrojó gases a los manifestantes. & Represión \\
& HM1. La policía les abrió las mochilas a los manifestantes. & $\begin{array}{l}\text { Represión } \\
\text { Cortesía }\end{array}$ \\
& HM2. La policía les abrió la puerta a los manifestantes. & Ayuda \\
& HC. Juan le cortó el pasto a la anciana. & Ayuda \\
3 & HM1. Juan le llevó los medicamentos a la anciana. & Molestia \\
& HM2. Juan le llevó un perro de la calle a la anciana. & Seducción \\
& HC. El taxista le dio flores a la pasajera. & Seducción \\
5 & HM1. El taxista le dijo un poema a la pasajera. & Intercambio comercial \\
\hline
\end{tabular}

Nota HC = Hecho clave; $\mathrm{HM}=$ Hecho meta; $\mathrm{CRE}=$ Categoría relacional de esquema. Se destacan con cursiva las relaciones.

\section{RESULTADOS Y DISCUSIÓN}

Se realizó una prueba $t$ de Student para muestras relacionadas, con el propósito de determinar si la calidad de las analogías variaba en función de la pertenencia de los hechos meta a una misma CRE que el hecho base. Este análisis reveló diferencias significativas entre las puntuaciones de calidad de la condición de pertenencia a una misma $C R E(M=4.41, D E=.208)$ y las puntuaciones de calidad de la condición de no pertenencia a una misma CRE $(M=1.25, D E=.131), t(17)=-51.79, p<.01$. La media de calidad obtenida en la condición de pertenencia a una misma CRE refleja que los participantes consideran entre "bastante" y "totalmente análogos" a estos hechos, mientras que la media obtenida en la condición de no pertenencia a una misma CRE muestra que los participantes consideran entre "nada" y "apenas análogos" estos hechos. Los resultados obtenidos evidencian que, a pesar de que los hechos comparados no se parezcan en sus relaciones, las personas tienden a considerarlos como análogos si constituyen ejemplares de una misma CRE. Por el contrario, las personas tienden a considerar como no análogos a dos hechos si estos no pertenecen a una misma CRE.

De manera complementaria, se realizó un análisis cualitativo de las justificaciones dadas por los participantes, con el propósito de confirmar si el criterio que habian empleado para determinar la calidad de las analogias era, tal como lo presuponía la manipulación experimental, el de la pertenencia o no a una misma CRE. Para ello se le proporcionaron a un juez independiente las justificaciones de los participantes (transcriptas en computadora e impresas en papel), acompañadas de una pregunta que debían responder por sî o por no. Las justificaciones correspondientes a las comparaciones cuyos análogos pertenecian a una misma CRE fueron presentadas al juez acompañadas de la siguiente pregunta: “¿Menciona el participante que los dos hechos son casos de la categoría represión o alguna similar?” (la palabra en itálica representa la $\mathrm{CRE}$ correspondiente a cada conjunto de materiales). Las justificaciones correspondientes a las comparaciones cuyos análogos no pertenecían a una misma CRE fueron presentadas al juez acompañadas de la siguiente pregunta: 
“¿Menciona el participante que uno de los hechos no es un caso de represión o de alguna categoría similar?”. El análisis de las justificaciones reveló que, en la condición de pertenencia, el 97\% de los participantes nombró la CRE crítica o una similar al justificar qué tenían en común los dos análogos. Por su parte, en la condición de no pertenencia, el $89 \%$ de los participantes nombró a la $\mathrm{CRE}$ crítica o una similar al justificar en qué diferían los análogos.

\section{EXPERIMENTO 2}

El objetivo de este segundo experimento consistió en evaluar la hipótesis de que la similitud de relaciones no es una condición suficiente para que dos hechos sean considerados análogos, ya que si dos hechos no constituyen ejemplares de una misma CRE no serán considerados de esa forma, a pesar de que sus relaciones se parezcan. Al igual que en el Experimento 1, los participantes recibieron un hecho base seguido de dos hechos meta, con la instrucción de determinar el grado en que cada hecho meta podía ser considerado análogo al hecho base. Sin embargo, a diferencia del Experimento 1 , en los materiales del presente experimento ambos hechos meta se parecian al base por lo que respecta a sus relaciones. Más allá de que los dos hechos meta se parecían en cuanto a la relación al hecho base, solo un hecho meta pertenecía a la misma CRE del base. Por ejemplo, en uno de los conjuntos de materiales el hecho base era "La policía arrojó gases a los manifestantes". Uno de los hechos meta de este conjunto era "La policía lanzó perros a los manifestantes". El objeto al que se aplica la acción descrita por la relación lanzar (perros) lo convierte en un ejemplar de la CRE represión, aplicable también al hecho base. En contraposición, el objeto del hecho meta restante determinaba que este fuera un ejemplar de una CRE alternativa. En este conjunto, el hecho meta era "La policía lanzó flores a los manifestantes", un caso de la CRE acercamiento amistoso. La predicción que se sigue de la teoría de la proyección de la estructura es que los participantes considerarian que ambos hechos meta son igualmente análogos al hecho base, dada la similitud entre las relaciones base y meta. En cambio, la predicción del enfoque categorial es que, más allá de que haya parecido entre las relaciones base y meta, las personas considerarian análogos a los pares cuyos hechos pertenecen a una misma CRE y no en cambio a los pares cuyos hechos pertenecen a CREs diferentes. Si se dieran estos últimos resultados, se contaria con una prueba de que dos hechos pueden no ser considerados análogos a pesar de que mantienen relaciones similares, siendo clave para que sí lo sean que la interacción que mantiene la relación y los demás componentes de la situación resulten, en cada uno de los hechos, en ejemplares de una misma CRE.

\section{MÉTODO}

\section{Participantes}

Formaron parte de este estudio 18 estudiantes de Psicología de la Universidad Nacional del Comahue $(M=19.93$ años; $D E=2.84)$. Todos los participantes firmaron un consentimiento informado antes de comenzar la experiencia. 


\section{Diseño y procedimiento}

El diseño y procedimiento fueron idénticos a los del Experimento 1.

\section{Materiales}

Los materiales fueron idénticos en todo a los del Experimento 1, salvo en que lo que respecta al parecido de las relaciones base y meta. Con el propósito de mostrar que el parecido de relaciones no es necesario para considerar que dos hechos son análogos, en el Experimento 1 se emplearon hechos meta cuyas relaciones no eran similares a las relaciones base. En cambio, dado que en el presente experimento se buscó mostrar que el parecido de relaciones no es suficiente para considerar que dos hechos son análogos, los dos hechos meta incluyeron relaciones similares a las del hecho base. Se construyeron seis conjuntos de materiales, cada uno de los cuales estuvo conformado por un hecho base y dos hechos meta. Uno de los conjuntos de materiales fue el siguiente:

Hecho clave. Juan le cortó el pasto a la señora.

Hecho meta 1. Juan le arrancó la aguaviva a la señora.

Hecho meta 2. Juan le arrancó el pelo a la señora.

Tal como lo ilustra este conjunto, los dos hechos meta incluian relaciones idénticas entre sí (arrancar) y similares a las de hecho base (cortar). Los hechos meta no eran similares con respecto al base ni entre sí por lo que respecta al objeto al que se aplicaba la acción. Mientras que en uno de los hechos meta el objeto al que se aplicaba la acción descrita por la relación (aguaviva) lo convertía en un ejemplar de la CRE que es aplicable al hecho base (ayuda), en el segundo no lo hacía (pelo). Los materiales tenían una estructura proposicional RELACION (agente, objeto, paciente) y los agentes y pacientes se mantenían constantes en los tres hechos. En la Tabla 2 se presentan tres conjuntos de materiales de los seis empleados. El orden de presentación de los materiales y la ubicación derecha-izquierda de los dos hechos meta fue decidido por azar simple para cada participante. El grado de similitud de las relaciones y entidades incluidas en los materiales de este experimento fue determinado, al igual que en el Experimento 1, por un grupo independiente de 20 participantes (extraídos de la misma población que los participantes experimentales). Se les pidió que puntuaran la similitud entre los elementos base y meta (e.g., la similitud entre cortar y arrancar). Este grupo recibió una lista con pares de conceptos, algunos de los cuales eran pares de relaciones y otros pares de objetos, provenientes de un conjunto inicial de materiales. Debían emitir un juicio de similitud a través de una escala Likert de cinco puntos: 1 (No similares), 2 (Apenas similares), 3 (Medianamente similares), 4 (Muy similares), 5 (Totalmente similares). Se seleccionaron para formar parte de este experimento seis conjuntos de materiales cuyos pares de objetos obtuvieron una puntuación media de similitud inferior a 2, y cuyos pares de relaciones obtuvieron una media de similitud superior a 4. 
Tabla 2.

Ejemplos de conjuntos de materiales empleados en el Experimento 2.

\begin{tabular}{cll}
\hline $\begin{array}{c}\text { Conjunto } \\
\mathbf{N}^{\circ}\end{array}$ & \multicolumn{1}{c}{ Hechos clave y meta } & CRE \\
\hline \multirow{3}{*}{1} & HC. La policía les arrojó gases a los manifestantes. & Represión \\
& HM1. La policía les lanzó perros a los manifestantes. & Represión \\
& HM2. La policía les lanzó flores a los manifestantes. & Acercamiento amistoso \\
& HC. Juan le cortó el pasto a la señora. & Ayuda \\
3 & HM1. Juan le arrancó la aguaviva a la señora. & Ayuda \\
& HM2. Juan le arrancó el pelo a la señora. & Agresión \\
& HC. El taxista le dio flores a la pasajera. & Seducción \\
5 & HM1. El taxista le regaló un libro a la pasajera. & Seducción \\
& HM2. El taxista le regaló una tarjeta de la empresa a la pasajera. & Publicidad \\
\hline
\end{tabular}

Nota. $\mathrm{HC}=$ Hecho clave; $\mathrm{HM}=$ Hecho meta; $\mathrm{CRE}$ = Categoría relacional de esquema. Se destacan con cursiva las relaciones.

\section{RESULTADOS Y DISCUSIÓN}

Se realizó una prueba $t$ de Student para muestras relacionadas, con el propósito de determinar si la calidad de las analogias variaba en función de la pertenencia de los hechos meta a una misma CRE que el hecho base. Este análisis reveló diferencias significativas entre las puntuaciones de calidad en la condición de pertenencia a una misma $C R E(M=4.45, D E=.220)$ y las puntuaciones de calidad en la condición de no pertenencia a una misma CRE, $(M=1.38, D E=.205), t(17)=-40.83, p<.01$. La media de calidad obtenida en la condición de pertenencia a una misma CRE refleja que los participantes consideran entre "bastante" y "totalmente análogos" a estos hechos, mientras que la media obtenida en la condición de no pertenencia de los hechos a una misma CRE muestra que los participantes consideran entre "nada" y "apenas análogos" estos hechos. Los resultados obtenidos evidencian que, a pesar de que los hechos comparados se parezcan en sus relaciones, las personas tienden a considerarlos como análogos solo si constituyen ejemplares de una misma CRE. Por el contrario, las personas tienden a considerar como no análogos dos hechos cuyas relaciones son parecidas si estos no pertenecen a una misma CRE.

De manera complementaria, se realizó un análisis cualitativo de las justificaciones verbales con el propósito de indagar si el criterio empleado por los participantes para determinar la calidad de las analogías era, tal como lo presuponía la manipulación experimental, el de la pertenencia o no a una misma CRE. El procedimiento seguido para el análisis de las justificaciones fue idéntico al empleado en el Experimento 1. Se halló que en la condición de pertenencia el 93\% de los participantes nombró la CRE crítica o una similar al justificar qué tenían en común los dos análogos. Por su parte, en la condición de no pertenencia el $84 \%$ de los participantes nombró a la CRE crítica o una similar al justificar en qué diferían los análogos. El resultado de este análisis cualitativo constituye una evidencia complementaria del hecho de que, a pesar de que las relaciones base y meta sean parecidas, las personas tienden a considerar como análogos dos hechos solo si estos pertenecen a una misma 
CRE. En este sentido, la similitud de relaciones parece no ser una condición suficiente para que dos hechos sean considerados análogos.

\section{DISCUSIÓN GENERAL}

En los dos experimentos del presente estudio los participantes consideraron más análogas a las comparaciones en las que los hechos pertenecian a una misma CRE que a las comparaciones en la que los hechos no pertenecían a una misma CRE. En el marco del Experimento 1, esta diferencia en las puntuaciones de calidad permitió determinar que, cuando los hechos comparados no se parecen en sus relaciones, las personas tienden a considerarlos como análogos si constituyen ejemplares de una misma CRE, y como no análogos, si no pertenecen a una misma CRE. En el marco del Experimento 2, la diferencia en las puntuaciones de calidad permitió determinar que, cuando los hechos comparados se parecen en sus relaciones, las personas tienden a considerarlos análogos solo si, además, ambos hechos pertenecen a una misma CRE. Por el contrario, cuando los hechos comparados poseen relaciones similares, pero no pertenecen a una misma $\mathrm{CRE}$, las personas no los consideran como análogos.

En conjunto, estos resultados constituyen evidencia de que la similitud de relaciones no es una condición necesaria (Experimento 1) ni suficiente (Experimento 2) para que dos hechos sean considerados como análogos, y que lo que determina que dos situaciones sean consideradas análogas es su pertenencia a una misma CRE. Estos resultados parecen contradecir una de las afirmaciones centrales de la teoría de la proyección de la estructura, a saber, que el componente central en la realización de una analogía consiste en detectar que dos situaciones comparten un sistema de relaciones similares, condición definitoria (i.e., necesaria y suficiente) para establecer que algo es o no es una analogía. Sin embargo, la teoría de la proyección de la estructura podría argumentar que la maquinaria analógica no fue pensada para lidiar con la actividad de comprender los análogos, sino para comenzar a operar una vez que los análogos han sido completamente comprendidos (véase, e.g., Morrison y Dietrich, 1995). En este sentido, los hechos "El taxista le dio un perfume a la pasajera" y "El taxista le escribió un poema a la pasajera" podrían ingresar en el "módulo" analógico una vez representados como dos instancias de seducir, o como dos relaciones similares (e.g., cortejar y seducir), de manera que los mecanismos re-representacionales estándares puedan operar para detectar la similitud relacional entre los dos hechos. Desde nuestro punto de vista, tal equipamiento analógico sería bastante limitado en sus posibilidades. Un primer problema se refiere al hecho de que el proceso de comparación no culmina en la detección de similitudes (e.g., "estos son dos casos de seducir"), sino que continúa para determinar diferencias en las dimensiones críticas de los hechos comparados (e.g., "esta seducción es más romántica que esta otra"), algo que solo podría ser llevado a cabo considerando las representaciones más específicas de cada uno de los análogos. Una segunda limitación consiste en que tal módulo no funcionaría para aquellos casos en los que las representaciones requeridas no son el input del proceso de comparación, sino que son en cambio un efecto de este. Por ejemplo, un análogo meta que afirmase "El taxista compartió sus problemas sentimentales con su pasajera" podría ser interpretado como un intento de seducción en el contexto de una comparación analógica, pero no cuando se lo considera de manera aislada (para evidencia empírica, véase Oberholzer et al., 2011). Un 
problema final con dicho módulo analógico se refiere al hecho de que hay casos en los que el significado de una CRE no puede ser completamente descripto por una relación o un sistema de relaciones. Muchas CREs están de hecho lexicalizadas como sustantivos representando entidades y no poseen un verbo correspondiente representando una relación (e.g., "fraude"). Podría argumentarse que esto representa un hecho lingüistico accidental, dado que no es dificil imaginar el posible surgimiento del verbo "fraudear". El gran problema es que muchas CREs parecen referir a estructuras complejas que resultan dificiles de conceptualizar exclusivamente en términos de un sistema de relaciones. Para ilustrar con un ejemplo coloquial, en Argentina algunas personas emplean la expresión "provisorio para siempre" para referirse a situaciones en las que una persona resuelve un problema doméstico de una manera informal y subóptima, creyendo erróneamente que contratará más adelante a un experto, quien proveerá la solución apropiada y definitiva. Resulta dificil imaginar una manera de describir esta categoría exclusivamente en términos de un sistema de relaciones, y sin hacer ninguna referencia, por ejemplo, a las propiedades de las soluciones generadas (para una miriada de ejemplos de este tipo, ver Hofstadter \& Sander, 2013). Quizás sólo se trate de que no somos lo suficientemente inteligentes para identificar el sistema de relaciones apropiado, o, nuevamente, de que lenguaje particular no incluye actualmente las palabras que son necesarias para describirlo. Esto podria ser cierto, pero atañe a alguien que adscribe a la visión de la proyección de la estructura explicar por qué muchas veces fallamos al detectar o describir verbalmente estos sistemas. Desde nuestro punto de vista, el camino adecuado para avanzar en una correcta conceptualización de qué es una analogia, consiste en reabrir la discusión con respecto al concepto de relación, no reduciéndolo a la fórmula sintáctica de "predicado de dos o más argumentos" y ampliarlo al de "estructura relacional". Con este concepto nos referimos al hecho de que, en una situación, todos los roles temáticos y sus vínculos (e.g., el vínculo entre acción y objeto, el vínculo entre agente y paciente) tienen importancia, y que lo que cuenta de los llenadores de dichos roles son las propiedades que se vuelven relevantes solo en el marco de esos vínculos (e.g., en una CRE como seducción, del objeto flores no interesa que es una parte de una planta, sino el valor romántico que adquiere al aplicársele la acción dar, en el marco de una relación agente-paciente apropiada para la categoria).

Si bien en este trabajo se empleó el enfoque categorial para explicar en qué medida dos hechos son juzgados como análogos, es preciso destacar que dicho enfoque tiene también implicaciones para otros subprocesos del razonamiento analógico. Por ejemplo, en relación con la formulación de inferencias, Minervino, Margni, Tavernini y Trench (2019) han demostrado que esta parece estar basada no simplemente en la copia y transferencia de relaciones base hacia el AM (tal como lo postula el enfoque estándar, e.g., Falkenhainer et al., 1989), sino en la búsqueda de ejemplares en el dominio meta de la categoría relacional a la que pertenece la información base a ser proyectada. En otro estudio, Olguín, Tavernini, Pacella y Minervino (2017) mostraron que, en ocasiones, la recuperación analógica no está basada en la similitud en aspectos generales entre los elementos base y meta (e.g., en el parecido de relaciones u objetos), tal como lo sostiene el enfoque estándar (para una revisión, véase Trench y Minervino, 2017), sino que está determinada por las similitudes en los valores que las situaciones base y meta presentan en una dimensión de la CRE a la que pertenecen. Por otra parte, 
Oberholzer, Trench, Kurtz y Minervino (2018) demostraron que, si un análogo base típico de una CRE es presentado previamente a un análogo meta atípico de esa misma CRE, las personas podrían ver estos hechos como análogos en virtud de un proceso de re-representación del meta en términos de la CRE base, incluso si las relaciones son diferentes. Este estudio muestra que, a pesar de que los hechos base y meta no se parezcan en sus relaciones, y que, inicialmente, tampoco pertenezcan a una misma CRE, el mismo proceso de comparación podría hacer que las personas modifiquen la representación de uno de los hechos para verlos, finalmente, como análogos. Futuras investigaciones podrian orientarse a avanzar en el estudio de las estructuras de las CREs y sus relaciones con el pensamiento analógico.

\section{REFERENCIAS}

Clement, C. A., \& Gentner, D. (1991). Systematicity as a selection constraint in analogical mapping. Cognitive science, 15, 89-132. https://doi.org/10.1207/s15516709cog1501_3

de la Fuente, J., \& Minervino, R. A. (2008). Pensamiento analógico. En M. Carretero \& M. Asensio (Eds.), Psicología del pensamiento: Teoría y prácticas (pp. 247-268). Madrid: Alianza Editorial.

Falkenhainer, B. (1990). Analogical interpretation in context. En M. Piatelli Palmarini (Ed.), Proceedings of the 12th Annual Conference of the Cognitive Science Society (pp. 69-76). Hillsdale, NJ: Lawrence Erlbaum Associates.

Falkenhainer, B., Forbus, K. D., \& Gentner, D. (1989). The structure-mapping engine: Algorithm and examples. Artificial Intelligence, 41, 1-63. https://doi.org/10.1016/0004-3702(89)90077-5

Forbus, K. D. \& Gentner, D. (1989, julio). Structural evaluation of analogies: What counts. En Proceedings of the Eleventh Annual Conference of the Cognitive Science Society (Vol. 34, pp. 341-348). Hillsdale, NJ: Lawrence Erlbaum Associates.

Gentner, D. (1983). Structure-mapping: A theoretical framework for analogy. Cognitive Science, 7, 155-170. https://doi.org/10.1207/s15516709cog0702_3

Gentner, D. (1989). The mechanisms of analogical transfer. En S. Vosniadou \& A. Ortony (Eds.), Similarity and Analogical Reasoning (pp. 199-241). Cambridge, UK: Cambridge University Press. https://doi.org/10.1017/CBO9780511529863.011

Gentner, D. (2003). Why we're so smart. En D. Gentner \& S. Goldin-Meadow (Eds.), Language in mind: Advances in the study of language and thought (pp. 195-235). Cambridge, MA: MIT Press. https://doi.org/10.7551/mitpress/4117.003.0015

Gentner, D. \& Kurtz, K. (2005). Relational categories. En W. K. Ahn, R. L. Goldstone, B. C. Love, A. B. Markman \& P. W. Wolff (Eds.), Categorization inside and outside the lab (pp. 151-175). Washington, DC: APA. https://doi.org/10.1037/11156-009

Gentner, D. \& Maravilla, F. (2018). Analogical reasoning. En. L. J. Ball \& V. A. Thompson (Eds.) International Handbook of Thinking \& Reasoning (pp. 186-203). Nueva York, NY: Psychology Press.

Gentner, D. \& Markman, A. B. (1997). Structure mapping in analogy and similarity. American Psychologist, 52, 45-56. https://doi.org/10.1037/0003-066X.52.1.45

Gentner, D. \& Markman, A. B. (2005). Defining structural similarity. Journal of Cognitive Science, 6, 1-20.

Gentner, D., Rattermann, M. J., \& Forbus, K. D. (1993). The roles of similarity in transfer: Separating retrievability from inferential soundness. Cognitive Psychology, 25, 431-467. https://doi.org/10.1006/cogp.1993.1013

Goldwater, M. B., Markman, A. B., \& Stilwell, C. H. (2011). The empirical case for role-governed categories. Cognition, 118, 359-376. https://doi.org/10.1016/j.cognition.2010.10.009

Goswami, U. (2001). Analogical reasoning in children. En D. Gentner, K. J. Holyoak, \& B. N. Kokinov (Eds.), The analogical mind. Perspectives from Cognitive Science (pp. 437-470). Cambridge: The MIT Press.

Hofstadter, D. R., \& Sander, E. (2013). Surfaces and essences: Analogy as the fuel and fire of thinking. New York, NY: Basic Books.

Holyoak, K. J. (2012). Analogy and relational reasoning. En K. J. Holyoak \& R. G. Morrison (Eds.), The Oxford handbook of thinking and reasoning (pp. 234-259). New York, NY: Oxford University Press. https://doi.org/10.1093/oxfordhb/9780199734689.001.0001

Holyoak, K. J., \& Thagard, P. R. (1989). Analogical mapping by constraint satisfaction. Cognitive Science, 13, 295-355. https://doi.org/10.1207/s15516709cog1303_1

Holyoak, K. J., \& Thagard, P. R. (1995). Mental leaps: Analogy in creative thought. Cambridge, MA: The MIT Press.

Hummel, J. E., \& Holyoak, K. J. (1997). Distributed representations of structure: A theory of analogical access and mapping. Psychological Review, 104, 427-466. https://doi.org/10.1037/0033295X.104.3.427

Inhelder, B., \& Piaget, J. (1955). De la logique de l'enfant à la logique de l'adolescent. París: Presses Universitaires de France.

Markman, A. B., \& Stilwell, C. H. (2001). Role-governed categories. Journal of Experimental and Theoretical Intelligence, 13, 329-358. https://doi.org/10.1080/09528130110100252

Minervino, R. A., Margni, A., Tavernini, L. M., \& Trench, M. (2019). The role of schema-governed categories in 
Tavernini, L. M. \& Minervino, R. A. / Psicodebate, 19(1), 62 - 76.

analogical inference. Manuscrito en preparación.

Minervino, R. A., Oberholzer, N., \& Trench, M. (2008). Similarity between propositional elements does not always determine judgments of analogical relatedness. En B. C. Love, K. McRae, \& V. M. Sloutsky (Eds.), Proceedings of the 30th Annual Conference of the Cognitive Science Society (pp. 91-96). Austin, TX: Cognitive Science Society.

Minervino, R. A., Oberholzer, N., \& Trench, M. (2013). Global Similarity Overrides Element Similarity when Evaluating the Quality of Analogies. Journal of Cognitive Science, 14, 287-317. https://doi.org/10.17791/jcs.2013.14.3.287

Minervino, R. A, Tavernini, L. M., Gomez, A. E., \& Trench, M. (julio, 2017). The effect of differences detected during mapping on inference generation: The case of analogies framed by schema-governed categories. Trabajo presentado en la 4ta Conferencia Internacional sobre Razonamiento Analógico, París.

Morrison, C. \& Dietrich, E. (1995). Structure-mapping vs. High-level perception: The mistaken fight over the explanation of analogy. En J. D. Moore \& J. Fain Lehman (Eds.), Proceedings of the Seventeenth Annual Conference of the Cognitive Science Society (pp. 678-682). Hillsdale, NJ: Erlbaum.

Oberholzer, N., Trench, M., Kurtz, K. J., \& Minervino, R. (2018). Analogies without commonalities? Evidence of re-representation via relational category activation. Frontiers in Psychology, 9, 2441. https://doi.org/10.3389/fpsyg.2018.02441

Oberholzer, N., Trench, M., \& Minervino, R. A. (2011). When lighting a candle becomes a superstition: Analogical recategorization through the application of relational categories. En L. Carlson, C. Hoelscher, \& T. F. Shipley (Eds.), Proceedings of the 33 ${ }^{\text {rd }}$ Annual Meeting of the Cognitive Science Society (pp. 568-573). Austin, TX: Cognitive Science Society.

Olguín, M. V., Tavernini, L. M., Pacella, L., \& Minervino, R. A. (julio, 2017). Analogical retrieval mediated by everyday schema-governed categories. Trabajo presentado en la 4ta Conferencia Internacional sobre Razonamiento Analógico, París.

Piaget, J. (1957). Logique et equilibre dans les comportements du sujet. En L. Apostel, B. Mandelbrot, \& J. Piaget (Eds.), Etudes d'epistemologie genetique: Vol. 2. Logique et equilibre (pp. 27-117). Paris: Presses Universitaires de France.

Raven, J. C., Raven, J. C., \& Court, J. H. (1962). Advanced progressive matrices. London: HK Lewis.

Richland, L. E., Morrison, R. G., \& Holyoak, K. J. (2006). Children's development of analogical reasoning: Insights from scene analogy problems. Journal of experimental child psychology, 94, 249-273. https://doi.org/10.1016/j.jecp.2006.02.002

Sternberg, R. J. \& Nigro, G. (1980). Developmental patterns in the solution of verbal analogies. Child Development, 51, 27-38. https://doi.org/10.2307/1129586

Tavernini, L.M., Martín, A., \& Minervino, R. A. (2017). La teoría de la asignación categorial sobre el pensamiento analógico: un desafio al enfoque estándar. Psiencia, 9, 94.

Tavernini, L. M., Trench, M., Olguín, M. V., \& Minervino, R. A. (2018). El papel de la similitud de objetos en el pensamiento analógico: el enfoque de la asignación categorial. Subjetividad y procesos cognitivos, 22(1), 147-165.

Trench, M., \& Minervino, R. A. (2017). Cracking the problem of inert knowledge: Portable strategies to access distant analogs from memory. En B. H. Ross (Ed.), Psychology of Learning and Motivation (Vol. 66, pp. 1-41). Cambridge, MA: Academic Press.

Yan, J., Forbus, K., \& Gentner, D. (2003). A theory of rerepresentation in analogical matching. En R. Alterman \& D. Kirsh (Eds.), Proceedings of the 25th Annual Conference of the Cognitive Science Society (pp. 1265-1270). Mahwah, NJ: Cognitive Science Society. https://doi.org/10.21236/ADA466013

Recibido 26-06-2018 | Aceptado 19-07-2018 